\section{L'Actualité économique}

L'ACTUALITÉ

ÉCONOMIQUE

\section{La théorie de l'entrepreneur chez Jean-Baptiste Say et la tradition Cantillon-Knight}

\section{Philippe Steiner}

Volume 73, numéro 4, décembre 1997

URI : https://id.erudit.org/iderudit/602243ar

DOI : https://doi.org/10.7202/602243ar

Aller au sommaire du numéro

\section{Éditeur(s)}

HEC Montréal

ISSN

0001-771X (imprimé)

1710-3991 (numérique)

Découvrir la revue

Citer cet article

Steiner, P. (1997). La théorie de l'entrepreneur chez Jean-Baptiste Say et la tradition Cantillon-Knight. L'Actualité économique, 73(4), 611-627.

https://doi.org/10.7202/602243ar
Ce document est protégé par la loi sur le droit d'auteur. L'utilisation des services d'Érudit (y compris la reproduction) est assujettie à sa politique d'utilisation que vous pouvez consulter en ligne.

https://apropos.erudit.org/fr/usagers/politique-dutilisation/ 


\title{
LA THÉORIE DE L'ENTREPRENEUR CHEZ JEAN-BAPTISTE SAY ET LA TRADITION CANTILLON-KNIGHT
}

\author{
Philippe STEINER \\ ENS Fontenay / Saint-Cloud et \\ Université de Paris - Dauphine
}

\begin{abstract}
" Chaque grand homme exerce une force à retardement : toute l'histoire à cause de lui est reconsidérée et des milliers de secrets du passé se glissent hors de leurs cachettes et s'exposent à son soleil. On ne saurait prévoir tout ce qui un jour sera encore de l'histoire. Peut-être le passé reste-t-il encore essentiellement voilé. Tant de forces à retardement sont encore nécessaires! » Nietzsche, Le Gai savoir $(\mathrm{I}, 34)$
\end{abstract}

\section{INTRODUCTION}

Parmi les rares titres de gloire que Jean-Baptiste Say conserve par-devers lui, suite à l'effondrement de sa renommée après le $\mathrm{XIX}^{\mathrm{e}}$ siècle $\mathrm{l}^{\mathrm{l}}$, figure le fait qu'il est le seul, dans l'école classique, à mettre l'accent sur le rôle décisif de l'entrepreneur. Autant on a pu dire que Say ne comprenait pas les enjeux théoriques des débats (sur la rente, notamment) entre les économistes anglais, autant on peut dire, suivant en cela J. Schumpeter (1954 : 556-557), que Say reste isolé dans son insistance sur le rôle de l'entrepreneur. Pour une fois, Say se trouve dans la position de n'avoir pas été suivi et peut-être même pas compris.

Les infortunes de la position de Say ne s'arrêtent pourtant pas là. Lorsqu'il est question de sa théorie de l'entrepreneur, on la réduit le plus souvent à une conception de l'entrepreneur entendu comme gestionnaire de la production. Dans ce cadre, l'entrepreneur a essentiellement pour tâche de combiner au mieux les services producteurs qu'il loue sur les différents marchés ${ }^{2}$. Cette conception est

1. Une première version de ce texte a été présentée dans le cadre de la History of Economics Society (Babson College) puis au Séminaire d'Histoire de la Pensée Économique du Pr. A. Lapidus (Paris). Il a bénéficié des remarques et suggestions de différents participants et je remercie tout particulièrement Humberto Baretto, Gilbert Faccarello, Philippe Fontaine, Jean-Jacques Gislain, Bob Hebert et André Lapidus.

2. Lorsqu'on ne néglige pas l'apport de Say comme c'est le cas de J. Schumpeter (1911) ou de F. Knight (1921) c'est ce que l'on en retient ( $c f$. R.F. Hebert et A.N. Link, 1988 ou H. Baretto, 1989); l'exception majeure à cette interprétation réductrice est fournie par G. Koolman (1971). 
alors en contraste prononcé avec ce que l'on peut appeler la « tradition CantillonKnight » où l'entrepreneur est essentiellement défini par rapport à un tout autre problème, le problème de l'incertitude (R.F. Hebert et A.N. Link, $1988: 22$ et 71-72; H. Baretto, 1989 : 33-34).

L'objectif de cet article est de montrer que l'interprétation habituelle de la conception que Say propose de l'entrepreneur est incomplète car elle repose trop exclusivement sur l'étude de sa théorie de la production. Il convient, selon nous, de compléter cette approche par l'étude de sa théorie de la répartition où se trouvent de très solides éléments analytiques qui permettent de placer la conception de l'entrepreneur de Say dans la tradition Cantillon-Knight.

\section{LA THÉORIE DE LA RÉPARTITION DE J.-B. SAY}

Say se veut un continuateur de l'oeuvre de Smith; cette position est notoirement réaffirmée lorsque, après la mort de Ricardo, il s'oppose de plus en plus résolument à l'école ricardienne. Mais, en même temps, Say entretient un rapport critique à Smith notamment lorsqu'il est question de la différence entre les profits de l'entrepreneur et les profits du capital; une note de Say sur son exemplaire de la Wealth of Nations l'indique : «Smith s'est jeté dans un grand embarras faute d'avoir séparé en deux parties ce qu'il appelle profits $d u$ fonds. Il y a dans cette valeur deux éléments qu'il a distingués ailleurs sans pousser cette distinction dans le reste de son ouvrage. Ces deux éléments sont le profit de l'industrie ou si l'on veut le salaire du travail et l'intérêt du capital. Pourquoi vouloir chercher la valeur de l'un d'après la valeur de l'autre » (Say dans Hashimoto, 1980 : 71). La formulation que Say emploie dans la deuxième édition du Traité, lorsqu'il est question de la théorie de la répartition, généralise la remarque initiale : " Il (Smith) n'offre rien de complet, rien de bien lié sur la manière dont les richesses se distribuent dans la société, et je remarquerai que cette partie de l'économie politique offrait un champ presque vierge à défricher » (Say, 1814 (I) : lv).

Qu'en est-il plus précisément de sa contribution dans ce domaine ? La première idée tient dans la distinction entre les ressources (capital, industrie, terre) qui sont des stocks et les services producteurs tirés de ces ressources qui sont des flux. Chacun de ces services apparaît sur un marché particulier. Comme on peut distinguer, dans une première approximation, trois marchés pour l'industrie (avec l'industrie du savant, celle de l'entrepreneur et celle de l'ouvrier), on a 
donc cinq marchés de services producteurs à considérer. La deuxième idée tient au fait qu'un type théorique particulier, l'entrepreneur, est chargé de faire le lien entre ces marchés ${ }^{3}$.

Selon Say, et cela le particularise si on le rapporte à $\mathrm{Smith}^{4}$ ou, a fortiori, à Ricardo, les prix des services sont fixés comme n'importe quel autre prix : « Les raisons qui déterminent la valeur des choses, et qui agissent de la manière indiquée dans les chapitres précédents, s'appliquent indifféremment à toutes les choses qui ont une valeur, même aux plus fugitives; elles s'appliquent par conséquent aux services productifs que rendent l'industrie, les capitaux et les terres dans l'acte de production » (Say, 1814 (II) : 45, 3nl et 74) ${ }^{5}$. Say décline ensuite cette thèse générale en l'appliquant à chacun des marchés de services. Il n'étudie pas, à la manière de Walras, l'interaction existant entre les marchés pour former l'équilibre général de production; Say étudie ce qui lui semble être les conditions structurelles de l'offre et de la demande sur ces marchés et, à ce titre, il s'apparente plutôt à Walras lorsque ce dernier considère plus particulièrement le marché du service foncier dans le cas d'une société progressive.

Marché des services des savants. Les profits que retirent les savants du service qu'ils rendent à la production sont faibles en raison de la relation structurelle entre l'offre et la demande de tels services. Say aborde la question en termes de diffusion des connaissances et non en termes de découverte : le savant met sur le marché une offre de connaissances presque infinie en publiant ses découvertes sous forme de livre ou en les rendant publiques au travers de ses

3. Dans son Épitomé, Say met unilatéralement l'accent sur la seule fonction de coordination des tâches : « Entrepreneurs d'industrie. Ils concourent à la production en appliquant les connaissances acquises, le service des capitaux et celui des agents naturels, à la confection des produits auxquels les hommes attachent une valeur » (Say, 1819 (II) : 469). Et on comprend que cela ait pu orienter les commentateurs à considérer seulement cet aspect des choses. Toutefois, dans le corps du texte, Say retient de nombreuses qualités nécessaires à la fonction d'entrepreneur; celles-ci sont de deux ordres différents. On peut en ranger certaines sous le registre de la gestion de la production («il s'agit de mettre en jeu quelquefois un très grand nombre d'individus; il faut acheter ou faire acheter des matières premières, réunir des ouvriers, chercher des consommateurs, avoir un esprit d'ordre et d'économie ») et d'autres sous le registre de l'incertitude associée à l'activité marchande (« jugement, apprécier l'importance de telle production et le besoin qu'on en aura, obstacles à surmonter, inquiétudes à vaincre ») (Say, 1814 (II) : 75).

4. Sans rentrer dans les détails, la dérive théorique de Say par rapport à Smith s'explique de la manière suivante. Refusant la théorie des prix naturels, Say interprète les taux naturels des salaires, profits et rente - taux pour lesquels des lois particulières sont mises en avant par Smith comme les déterminants des niveaux de l'offre et de la demande pour chacun des services correspondants. Il retient seulement de Smith l'étape suivante la "gravitation » où règnent les lois de l'offre et de la demande : et, à ce moment-là, il se sent le véritable héritier de Smith.

5. Les formulations qui se trouvent dans une note du Traité (1814 (II) : 72-73) où Say déclare que les principes qui règlent les profits du capital et ceux de l'entrepreneur sont différents ne doivent pas en imposer. Les principes dont il est question sont ceux qui déterminent les niveaux de l'offre et de la demande sur chaque marché; ces principes ne se substituent pas, bien sûr, aux lois de l'offre et de la demande, mais ils explicitent les particularités de ces marchés. 
enseignements. La demande de ces connaissances est bien moindre, en conséquence de quoi les profits économiques du savant sont faibles (Say, 1814 (II) : $70-71)^{6}$.

Marché des services des entrepreneurs. Say insiste ici tout particulièrement sur les causes qui expliquent le faible niveau de l'offre des services des entrepreneurs d'industrie et de commerce. Trois éléments définissent la structure du marché : premièrement, n'étant pas nécessairement détenteur de capital, l'entrepreneur doit avoir le moyen de s'en procurer ce qui signifie qu'à titre de demandeur des services des capitaux, il doit être solvable et connu pour tel de la part des offreurs de capitaux (cf. P. Fontaine, 1993). Deuxièmement, les fonctions de l'entrepreneur en tant que gestionnaire de la production demandent que soient réunies des capacités qui ne le sont que rarement chez un même individu? ${ }^{7}$ Enfin, la fonction d'entrepreneur est inséparable d'un risque tel que les plus habiles peuvent y perdre honneur et fortune (ibid., pp. 75-76). L'offre des services des entrepreneurs est d'autant plus faible que la nature de l'activité productive demande la réunion de ces différentes conditions plus dans la manufacture et le commerce, moins dans l'agriculture.

Marché des services des ouvriers. Il s'agit du marché du travail à proprement parler et plus précisement du marché du travail déqualifié 8 . Les profits des ouvriers sont essentiellement déterminés par le fait que l'offre de service est toujours proportionnée à la demande qui en est faite (ibid., p. 79). L'argument employé est le suivant : le revenu nécessaire à la subsistance étant pris comme référence, Say considère que toute hausse des profits ouvriers, en raison du manque de prévoyance qui caractérise cette classe, se traduit par un accroissement de la population ouvrière et donc de l'offre du service correspondant. Les entrepreneurs sont donc ici dans une position particulière puisque, par l'intermédiaire de la loi de population, l'offre de ce service se proportionne - avec un temps de retard que Say néglige - à la demande qu'ils en font. Les profits ouvriers sont donc faibles.

6. En compensation, le savant est payé en prestige; en effet, Say avance l'idée que « l'honneur est une espèce de salaire qui fait partie des profits de certaines conditions » (ibid., p. 63).

7. Bien que cet aspect ne soit pas au centre du problème tel que nous l'envisageons ici, Say $y$ attache une grande importance. C'est un point sur lequel il insiste tout particulièrement dans son enseignement au Conservatoire : " Un des objets essentiels de ce cours, est de perfectionner la gestion des entreprises industrielles » (Say, 1828 : 157) et il poursuit : "Qu'est-ce donc qui fait la différence de succès entre une fabrique, un commerce qui prospèrent et d'autres qui ne vont pas bien ? La différence dans la gestion, dans l'administration de la chose (...) Cela s'apprend par l'expérience, dira-t-on, mais on n'acquiert l'expérience qu'à ses dépens (...) L'économie industrielle expérimentale, telle que je me propose de l'exposer devant vous, n'est que le résultat d'un grand nombre d'expériences, rangées suivant un ordre régulier où l'on a cherché à connaître, par le moyen de l'analyse, pourquoi telle cause a produit tel résultat » (ibid., p. 158).

8. « Je désigne ici, par le nom d'ouvrier, principalement celui qui travaille pour le compte d'un entrepreneur d'industrie (...). De plus je préviens que les ouvriers dont il est question dans ce paragraphe-ci sont ceux qui n'exigent point ou presque point d'étude » (ibid., p. 78nl). 
Marché des services des capitaux. Pour comprendre ce qui se passe sur ce marché, Say dissocie deux choses : la prime d'assurance que le capitaliste exige pour couvrir les risques de perte de son capital et le profit du capital correspondant à la rémunération des services rendus par ce service producteur (ibid., p. 95); c'est de ce dernier dont il est question ici ${ }^{9}$. Il est clair que la prise en compte simultanée des deux éléments est essentielle dans le rapport qu'entretiennent les entrepreneurs demandeurs de capitaux et les offreurs (les capitalistes) puisque ces deux éléments représentent pour l'entrepreneur le prix effectif qu'il paye pour disposer du service.

Sur ce marché, Say ne définit pas de situation structurelle généralement valable; on trouve plutôt une esquisse des nombreux éléments concourant à former l'offre et la demande sur ce marché. La demande de capitaux dépend de la demande qui est faite des produits fabriqués avec l'aide de ces capitaux. Cette dernière demande dépend elle-même de deux phénomènes : la demande agrégée varie avec le niveau de la richesse et sa hausse se répercute sur l'ensemble des secteurs à la différence de ce qu'il en est de la demande s'adressant à tel ou tel secteur. Avec la première, une demande accrue de biens se traduit par une demande plus forte de capitaux; avec la seconde, une demande accrue d'un bien peut être satisfaite par une réallocation des capitaux existant. L'offre de capitaux correspond aux capitaux actuellement disponibles qui cherchent à être prêtés. Cette offre dépend du degré de liquidité des capitaux existant et des motifs des capitalistes, c'est-à-dire de leur état d'esprit vis-à-vis de la sûreté du placement, de l'honnêteté et de la régularité de la politique économique du gouvernement, de l'état des moyens de communication ${ }^{10}$.

Marché des services des terres. Sur ce marché la demande de services dérive de la demande de biens agricoles; l'offre est donnée par la quantité de terres d'une certaine qualité existant sur le lieu. Ici, Say tient compte de la localisation, c'est-à-dire de la distance et des frais de transport afférents entre le lieu de production et le marché : « Les produits auxquels le fonds de terre a concourru lui rendent un profit proportionné à la part qu'il a eue à la production, moins les frais qu'il a fallu faire pour porter ces produits au marché, au lieu d'échange. Quand cette déduction ne laisse rien pour le profit du terrain, le terrain n'a aucun profit » (ibid., p. 126). Cela explique donc qu'il y ait des terres qui ne peuvent prétendre obtenir un profit pour le service qu'elles rendent et que seuls les propriétaires peuvent employer. Lorsque le profit foncier existe, si l'on tient compte du prix des terres, il se trouve dans une relation déterminée avec le profit du capital : le taux de profit du capital est comparable au taux de profit foncier ou rente (ibid., p. 128).

9. « Le capitaliste qui prête le capital vend le service, le travail de son instrument; le prix journalier ou annuel qu'un entrepreneur lui en paie se nomme intérêt. Les deux termes de l'échange sont, d'une part, le service du capital, et d'autre part l'intérêt » (Say, $1820: 454$ ).

10. Ce point est plus clairement élaboré dans le Cours complet (1828-1829 (II) : 70-71). 
Qu'en est-il maintenant des relations entre les divers marchés étudiés séparément ? Le texte de Say renferme un problème essentiel : quelles sont les relations entre les taux de rendement des différents services et, surtout, des différentes formes de capitaux ? Selon Say, il faut partir de l'idée que les ressources productives sont toutes caractérisées par une absence, plus ou moins prononcée, de mobilité; cela signifie que la formation d'un taux de rendement unique, ou d'une hiérarchie stable de ces taux de rendement suivant l'ampleur des risques encourus et de la durée de l'immobilisation du capital, n'est pas un processus clairement dégagé et étudié par Say. Il ne justifie guère cette prise de position, et il est difficile de penser qu'il s'agit de sa part d'une véritable hypothèse théorique; par ailleurs, il y a des remarques qui tendent à faire penser que les possesseurs de ressources, notamment les détenteurs de capitaux sous forme monétaire, placent leurs capitaux en fonction du taux de rendement attendu pour un risque donné ${ }^{11}$, mais cette perspective ne retient jamais véritablement son attention et il ne s'en empare pas pour faire progresser son analyse. Il se défie constamment de ce genre d'idées eu égard à l'usage qu'en fait Ricardo ${ }^{12}$ et il met en avant l'immobilité relative des capitaux (1814 (II) : 109-110; $1826: 403 ; 1828-1829$ (II) : 38, 48, 68-71), l'importance du jugement et de la perspicacité de l'entrepreneur (1814 (II) : 102; $1817: 135-136 ; 1821: 133 ; 1828-1829$ (II) : 36) ou bien il insiste sur les contraintes qu'impose la continuité des opérations productives : «Il y a des capitaux tellement engagés dans certaines entreprises, particulièrement dans les manufactures, que leurs entrepreneurs consentent souvent à en perdre les intérêts, à sacrifier les profits de leur propre industrie, et continuent à travailler uniquement pour soutenir l'entreprise jusqu'à des temps plus favorables, et pour n'en pas perdre le fonds : d'autres fois ils craignent de perdre de bons ouvriers que la suspension de l'ouvrage forcerait à se disperser » $(1820: 484)$.

Cet examen de la théorie de la répartition développée par Say montre bien la distance qui existe entre sa position et celle de Ricardo. Premièrement, Say s'oppose à l'idée ricardienne selon laquelle chaque variable a un niveau de marché qui gravite autour d'un niveau naturel et il construit sa problématique en se basant sur les seules lois de l'offre et de la demande. La différence porte alors sur la détermination des profits et sur la relation salaire-profit; il ne fait pas de

11. «Un capital qui ne rencontre pas dans une entreprise les avantages qu'il trouverait ailleurs, cherche un autre emploi » (ibid., p. 126); "Toutes les fois qu'on achète une terre avec un capital, ou un capital avec une terre, on est appelé à comparer la rente de l'une avec la rente de l'autre » (ibid., p. 128; la rente signifie chez Say le taux du profit du service); «Celui des deux qui se vendrait au-delà de ses frais de production attirerait vers sa production une partie des producteurs qui s'occupent de l'autre, jusqu'à ce que les services productifs fussent également payés dans l'un et dans l'autre genre : c'est un effet dont on convient généralement » (Say, $1820: 459)$.

12. «Ricardo soutient que tout emploi du capital est également avantageux au pays parce que s'il y avait un emploi qui rapportait plus que les autres, tous les capitaux s'y précipiteraient. Que les capitaux rapportent, tout balancé, des profits équivalents à leur possesseur, cela pourrait être vrai quoique fort contestable. Il est difficile de croire que les capitalistes qui ont (des) millions déposés en banque sans intérêts en tirent un profit égal au profit qu'un entrepreneur comme il y en a un immense nombre, retire de son capital » (Say, Mss, H : 393; cf. aussi 1826 : 403; 1828-1829 (II) : 68-71). 
doute, dans l'esprit de Say, que l'antagonisme ricardien en la matière n'est pas recevable. Deuxièmement, sans disconvenir qu'une égalisation des taux de rendement entre les divers placements puisse avoir lieu, Say met plutôt l'accent sur le fait de la disparité de ces taux. Troisièmement, Say ne dégage rien de comparable à la « dynamique grandiose » que Ricardo contruit sur la base de sa théorie de la répartition. Toutefois, cet examen montre que Say ne doit pas être perçu seulement comme un économiste qui n'aurait pas compris Ricardo. Il est plus intéressant de considérer Say comme un auteur qui s'attache à répondre à certaines questions qui ne sont pas celles de Ricardo. En d'autres termes, il faut maintenant examiner la façon dont Say développe sa pensée sur la théorie de la répartition pour constater la place importante qu'y occupe l'entrepreneur.

\section{LE RÔLE DE L'ENTREPRENEUR SELON LA THÉORIE DE LA RÉPARTITION}

Jusqu'à présent nous avons suivi Say sans mettre en question sa présentation et nous avons donc fait comme si le marché des entrepreneurs existait d'une façon non problématique. Il faut aller plus loin maintenant dans la mesure où, pour que ce marché existe, il faut pouvoir répondre à la question suivante : qui forme la demande sur ce marché ?

Conformément à la réflexion de l'auteur, nous avons retenu l'idée selon laquelle l'entrepreneur joue le rôle de demandeur sur le marché des services en faisant face aux détenteurs de ces services : les travailleurs, les savants, les capitalistes et les propriétaires fonciers. Mais qui demande le service des entrepreneurs ? L'entrepreneur lui-même ne peut tenir à la fois le rôle d'offreur et de demandeur. Ce marché existe-t-il alors ? Say n'est pas toujours précis sur ce point; il arrive même que certains textes passent sous silence le rôle tenu par les forces du marché dans la détermination des profits des entrepreneurs ${ }^{13}$. Mais l'essentiel de l'argumentation peut être maintenu si on opére une distinction entre le gestionnaire de la production et l'entrepreneur entendu comme un agent caractérisé par la vigilance et confronté à l'incertitude. Au moyen de cette distinction, présente à plusieurs reprises chez Say ${ }^{14}$, et soutenue par la structure théorique de son oeuvre, on peut maintenir l'idée d'un marché des services de l'entrepreneur (gestionnaire de la production) : ce type de service est demandé par l'entrepreneur proprement dit, et le prix du service est formé selon les lois

13. «Le profit de l'entrepreneur au compte duquel l'opération a été faite, en faisant abstraction de l'intérêt du capital qu'il peut y avoir employé, représente le salaire de son temps et de son talent, c'est-à-dire de ses propres services à lui-même » (Say, $1820: 450$ ).

14. «Dans quelle classe mettez-vous les banquiers, les courtiers, les commissionnaires en marchandises, qui travaillent pour compte d'autrui ? dans la classe des entrepreneurs parce qu'ils exercent leurs fonctions par entreprise, se chargeant de trouver les moyens d'exécution, et les employant à leurs frais » (Say, 1821 : 131). Un passage du Cours complet peut aussi être rapproché de ce type d'analyse. Say explique que certains salariés - il prend le cas des commissionnaires (Say, 1828-1829 (II) : 44) - d'une manufacture peuvent être considérés comme des entrepreneurs. Dans un tel cas, se trouve défini un marché pour ses services si l'on distingue ces entrepreneurs de l'entrepreneur vigilant qui se trouve au point de départ de l'entreprise (cf. I.M. Kirzner, 1973). 
générales de l'offre et de la demande, sous la forme qu'elles adoptent sur ce marché. Ce faisant, le problème du revenu de l'entrepreneur proprement dit apparaît en pleine lumière. Cela permet de donner un sens aux très intéressantes propositions, ignorées jusqu'à présent, qui placent sa conception de l'entrepreneur dans la lignée de ceux qui définissent l'entrepreneur par sa confrontation à l'incertitude ainsi que l'affirme, avec une particulière netteté, le passage suivant du Catéchisme : «Quelle est la première observation à faire sur les revenus des entrepreneurs d'industrie ? Qu'ils sont toujours variables et incertains (...)» (Say, $1821: 131)$.

Pour s'avancer dans une telle direction, il faut prêter attention au fait que Say développe une distinction qui se révèle d'une grande importance en matière de théorie de la répartition. Cette distinction trace une ligne de partage entre les revenus certains que les détenteurs des ressources, par l'intermédiaire d'une convention, obtiennent contre cession de leurs services et les profits que l'on retire, pour une période déterminée, sur des marchés concurrentiels de la location des services. Le prix du service est établi selon les rapports entre la demande et l'offre ${ }^{15}$. Est-ce que pour autant l'entrepreneur paye ces services à ces prix-là ? Aussi curieux que cela puisse paraître la réponse de Say est négative en raison de la distinction entre le prix des services et les revenus cédés (salaires, intérêt et fermage) : «La portion de la valeur produite que retire de cette façon le propriétaire foncier, s'appelle profits du fonds de terre; quelquefois il abandonne ce profit à un fermier moyennant un fermage. La portion retirée par le capitaliste, par celui qui fait les avances, quelques petites et quelques courtes qu'elles aient été, s'appelle profits du capital; quelquefois il prête son capital, et en abandonne le profit moyennant un intérêt. La portion retirée par les industrieux, se nomme les profits de l'industrie; quelquefois ils abandonnent ce profit moyennant un salaire » (Say, 1814 (II) : 49). Examinons ce point un peu plus en détail en nous appuyant, entre autre, sur un chapitre du Cours complet destiné à expliciter cette distinction.

Dans ce dernier texte, après avoir repris la distinction introduite dans le Traité entre les revenus cédés et les prix des services productifs, Say marque une

15. Say modifie assez sensiblement cette partie de son ouvrage suite aux discussions sur la théorie de la valeur avec Ricardo. On sait que la quatrième édition comporte des développements importants sur « la théorie des services producteurs » où Say avance des formulations proches de celles de la théorie de l'imputation : «C'est la valeur d'une multitude de produits balancés entre eux, qui fonde la valeur courante des services productifs, et ce n'est pas la valeur des services productifs qui fonde la valeur des produits, ainsi que l'ont avancé quelques auteurs; et comme c'est l'utilité du produit qui le fait rechercher, qui lui établit une valeur, c'est la faculté de pouvoir créer cette utilité qui fait rechercher les services productifs, qui leur établit une valeur; valeur qui équivaut pour chacun d'eux à l'importance de sa coopération, et dont le total forme, pour chaque produit, ce qu'on appelle ses frais de production " (Say, 1819 (II) : 9-10). Ces formulations sont à leur tour très profondément modifiées dans la cinquième édition du Traité (Say, $1826: 320-327$ ), ainsi que dans le Cours complet. C'est la raison pour laquelle l'argumentation de S. Hollander (1985) ne nous paraît pas recevable ( $c f$. aussi T. Peach, $1993: 263-267$ ) lorsque, s'appuyant seulement sur la quatrième édition du Traité, il laisse entendre qu'il existe une théorie de l'imputation chez Say. 
différence essentielle quant à la nature de ces deux sortes de revenus : "Celui qui fait ainsi l'acquisition des services productifs de la terre, du capital, ou de quelque autre faculté personnelle d'un autre, en paie un prix fixe et convenu, qui forme pour le vendeur un revenu certain et déterminé; et un revenu incertain et variable pour l'entrepreneur » (Say, 1828-1829 (II) : 27; je souligne). Il poursuit en accentuant le caractère d'incertitude associé à la fonction d'entrepreneur : « L'acquéreur à forfait d'un revenu, prend donc à son compte les chances favorables ou contraires qui peuvent rendre en réalité ce revenu plus ou moins considérable » (ibid.). Cette distinction est-elle purement formelle ou bien a-t-elle un contenu analytique précis dans la théorie de Say ? On peut répondre positivement sans hésitation.

Comment sont déterminés les revenus cédés ? Sémantiquement, c'est sans doute dans le Traité que la présentation est la plus claire car, lorsqu'il est question des revenus cédés, Say parle de convention entre le détenteur du service et l'entrepreneur (Say, 1814 (II) : 89, 98). Mais quel que soit l'ouvrage pris en référence, le fond théorique reste le même. Say marque la différence entre les deux sortes de revenu par le fait que les revenus cédés ne sont pas seulement expliqués par le rapport entre l'offre et de la demande, car les rapports de force entre les agents interviennent aussi. Ce qui distingue le fonctionnement du marché, basé sur les lois de l'offre et de la demande, et la formation de cette convention tient au fait que si la première donne un cadre anonyme et général dans lequel la seconde va prendre place, la convention fait intervenir les relations de pouvoir dans la détermination du montant des revenus cédés. Chaque marché (du travail, du capital et de la terre) est particulier sous ce registre; prenons l'exemple du dernier d'entre eux. Le fermier, dit Say, se trouve dans une très mauvaise position vis-à-vis du propriétaire qui ne cherche pas à exploiter lui-même sa terre. En effet, le fermier-entrepreneur est le seul à offrir un service qui ne soit pas relativement rare car il utilise des pratiques routinières plus que les autres entrepreneurs; par ailleurs, sur le marché de la location des terres, il se trouve confronté à une ressource naturellement limitée dont l'offre ne peut s'accroître. Finalement, dans la convention qui détermine le fermage du propriétaire, le fermier affronte une classe dont le pouvoir social est solidement implanté. Aussi, Say prend-il en compte ce rapport de force lorsqu'il étudie le fermage : "Outre l'avantage que le propriétaire tient de la nature des choses (Note de l'auteur : la quantité de terre est limitée), il en tire un autre de sa position qui d'ordinaire lui donne sur le fermier l'ascendant d'une fortune plus grande, et quelquefois celui du crédit et des places » (Say, 1814 (II) : 132). Le Cours complet s'avance d'une manière plus résolue dans ce sens : " partout on a attribué des avantages sociaux à la propriété des terres. Je ne parle pas des privilèges pécuniaires attachés en certains pays à la possession des terres nobles; mais de la prépondérance qui naît de l'exercice de certaines fonctions, comme celles d'électeurs et de députés, ou d'administrateurs, ou de juges, prépondérance qui, dans le marché qu'un propriétaire passe avec un fermier, donne au premier un certain avantage pour stipuler les clauses qui lui sont avantageuses, ou pour faire 
décider en sa faveur les litiges auxquels elles peuvent donner lieu » (Say, 18281829 (II) : 118). La conséquence économique d'une telle situation est loin d'être négligeable : le propriétaire occupe une position de monopole, dit Say, et le fermier-entrepreneur lui paye, à titre de revenu certain (le fermage), l'intégralité du profit du service.

La situation de cet entrepreneur-fermier est tout à fait particulière, mais elle fait apparaître, par contraste, la position dans laquelle se trouvent les autres entrepreneurs (manufacturiers, négociants, commerçants) vis-à-vis des possesseurs de ressources qui vendent leurs profits (incertains) contre un revenu certain. Dans les relations débouchant sur la convention entre l'entrepreneur et le possesseur de la ressource, Say inverse le sens du rapport de pouvoir en arguant du fait que, de par sa position d'intermédiaire entre les marchés et de gestionnaire de la production, l'entrepreneur possède des informations que les autres agents ne sont pas à même de réunir. Ces informations lui permettent d'établir au mieux de ses intérêts les conventions qu'il passe : « Il est bon de remarquer les autres avantages dont un chef d'entreprise, s'il est habile, peut tirer parti. Il est l'intermédiaire entre le capitaliste et le propriétaire foncier, entre le savant et l'ouvrier, entre toutes les classes de producteurs, et entre ceux-ci et le consommateur. Il administre l'oeuvre de la production; il est le centre de plusieurs rapports; il profite de ce que les autres savent et de ce qu'ils ignorent, et de tous les avantages accidentels de la production. C'est aussi dans cette classe de producteurs, quand les événements secondent leur habileté, que s'acquièrent presque toutes les grandes fortunes » (1814 (II) : 78). Si l'entrepreneur a bien calculé et si les événements lui sont favorables, la différence entre les profits initialement incertains et les revenus certains payés aux détenteurs des services qui ne veulent pas affronter l'incertitude, forme le revenu spécifique de l'entrepreneur ce que l'on appelle maintenant, le profit pur.

La logique de la théorie de la répartition de Say donne une réponse à la question posée plus haut à propos du marché des services des entrepreneurs. En distinguant l'entrepreneur proprement dit de l'entrepreneur gestionnaire de la production, on explique la formation d'une demande et d'une offre dont le prix du service sera le résultat. En faisant intervenir la distinction entre les revenus certains et les profits incertains, on a le moyen de rendre compte du profit pur qui revient à l'entrepreneur proprement dit. La théorie de l'entrepreneur de Say peut maintenant prendre place dans la tradition Cantillon-Knight.

\section{SAY Et la tradition CANTILlon-Knight}

La structure particulière de la théorie de la répartition de Say éclaire d'une manière véritablement nouvelle sa conception de l'entrepreneur. Dans le cadre de la théorie de la production, l'entrepreneur est plutôt présenté sous les traits d'un gestionnaire avisé qui combine au mieux les éléments nécessaires à l'accomplissement du processus de production. Cette fonction demeure dans le cadre de la répartition, mais elle ne représente plus qu'une facette du personnage 
qui est maintenant confronté à l'incertitude inhérente à toute opération économique. En mettant en évidence ce trait de la théorie de Say, il est possible de le faire entrer dans la filiation qui, de R. Cantillon à F.H. Knight, place précisément l'accent sur cet aspect des choses. Cette perspective demande à être brièvement précisée en considérant d'abord le rapport de Say à Cantillon, celui de Say à Knight ensuite.

Say fait peu de références à Cantillon, pour autant qu'il en fasse. Peut-être connaît-il son oeuvre par l'intermédiaire de Smith, des physiocrates ou de Germain Garnier; quoi qu'il en soit, son intérêt est faible pour ce domaine assez lointain de l'histoire de la science qui l'occupe ${ }^{16}$. Il est vrai que Say s'inscrit dans une perspective toute différente de celle de l'auteur de l'Essai sur la nature du commerce en général. Un siècle les sépare, et quel siècle! La structure sociale que décrivent leurs théories differe profondément : Cantillon conduit le raisonnement en posant « pour principe que les propriétaires de terres sont seuls indépendants naturellement dans un État; que tous les autres ordres sont dépendants, soit comme entrepreneurs, ou comme à gages, et que le troc et la circulation de l'État se conduit par l'entremise de ces entrepreneurs » (Cantillon, 1755 : $73)^{17}$. Il en va différemment chez Say : la structure sociale à laquelle il pense, est celle où le marché fournit à chaque intervenant son indépendance vis-à-vis des propriétaires fonciers, sinon vis-à-vis des propriétaires des capitaux ${ }^{18}$. Sur un plan analytique, les différences ne sont pas moindres. Cantillon ne développe pas une conception du « profit » facile à faire entrer dans le cadre de la théorie économique classique; il ne pose pas le problème de la coordination des différents marchés ${ }^{19}$. Même si Say utilise un vocabulaire qui peut surprendre et si, en comparaison des classiques anglais, il adopte une position originale, il appartient à une autre constellation théorique que celle de Cantillon : il isole les diverses catégories de revenus et indique les caractéristiques marchandes particulières qui concourent à leur formation.

16. Cantillon n'est mentionné ni dans les discours préliminaires des différentes éditions du Traité où Say brosse à grands traits l'histoire de l'économie politique moderne, ni dans l'appendice spécialement consacré à l'histoire de l'économie politique dans le Cours complet.

17. On se reportera au travail de H. Vérin (1982) pour l'éclairage qu'elle apporte sur l'environnement culturel d'où sort la conceptualisation de Cantillon.

18. Sa position change à ce propos comme on le remarque en comparant les différentes éditions du Traité (P. Steiner, 1997).

19. Voir les difficultés d'exégèses rencontrées sur ce point par les commentateurs récents (R. Prendergast, 1991; A. Brewer, 1993) et les conclusions prudentes - et justifiées nous semble-t-il de R. Prendergast, l'auteur qui s'avance le plus loin sur ce point. 
Le rappel de différences évidentes séparant les deux théories rend d'autant plus singulier le fait qu'elles se rejoignent sur un point central pour notre propos $^{20}$. Cantillon tient une part de son originalité du fait que l'entrepreneur est défini par l'incertitude, c'est-à-dire par le fait qu'il ne peut prévoir les états futurs des marchés ${ }^{21}$ et par la distinction associée entre des coûts certains et des revenus incertains ${ }^{22}$. Cette perspective disparaît complètement dans l'oeuvre de Quesnay ou de Turgot, comme dans les autres courants de la pensée économique de la fin du XVIII ${ }^{\mathrm{e}}$ siècle ${ }^{23}$; pourtant les deux caractéristiques de l'entrepreneur de Cantillon se retrouvent chez Say parmi l'ensemble des traits définissant la fonction remplie par l'entrepreneur.

Knight, de son côté, fait très peu de références à Say, alors que la traduction du Traité est largement diffusée aux États-Unis tout au long du XIX $\mathrm{X}^{\mathrm{e}}$ siècle. Là encore, il est évident qu'il existe une grande distance entre les deux auteurs - un autre siècle les sépare. Knight se trouve placé dans une conjoncture théorique et un état du développement économique différents de ceux de Say. Sur le premier point, Knight fait clairement entendre qu'il choisit une voie privilégiant la théorie ${ }^{24}$ et, ainsi, il adopte une problématique qui s'oppose à celle que Say présente en

20. On peut aussi relever que Say, comme Cantillon, mais pour d'autres raisons que lui, met en valeur le caractère décisif de la demande lorsqu'il s'agit de définir la structure de la production. C'est, par exemple, le cas dans le passage suivant : « Les besoins des consommateurs déterminent en tout pays les créations des producteurs. Le produit dont le besoin se fait le plus sentir, est le plus demandé; le plus demandé fournit à l'industrie, aux capitaux et aux terres de plus gros profits, qui déterminent l'emploi de ces moyens de production vers la création de ce produit » (Say, 1819 (II) : 217).

21. « Le fermier est un entrepreneur qui promet de payer au propriétaire, pour sa ferme ou terre, une somme fixe d'argent, sans avoir de certitude de l'avantage qu'il tirera de cette entreprise. Il emploie une partie de cette terre à nourrir des troupeaux, à produire du grain, du vin, des foins, etc. suivant ses idées, sans pouvoir prévoir laquelle des espèces de ces denrées rapportera le meilleur prix. Ce prix des denrées dépendra en partie des saisons et en partie de la consommation; s'il y a abondance de blé par rapport à la consommation, il sera à vil prix, s'il y a rareté, il sera cher. Qui est celui qui peut prévoir le nombre des naissances et morts des habitants d'un État, dans le courant de l'année ? Qui peut prévoir l'augmentation ou la diminution de dépense qui peut survenir dans les familles? Cependant le prix des denrées du fermier dépend naturellement de ces événements qu'il ne saurait prévoir, et par conséquent il conduit l'entreprise de sa ferme avec incertitude » (ibid., pp. 59-61; je souligne).

22. «(...) ils en donnent un prix certain suivant celui du lieu où ils achètent, pour les revendre en gros ou en détail à un prix incertain » (ibid., p. 53).

23. À titre de curiosité, on peut mentionner le débat suscité dans le Journal de Commerce en 1760 lorsqu'est posée la question : « Est-il licite, et jusqu'où est-il licite dans le commerce, de profiter des avis particuliers qu'on peut avoir ? ». L'une des réponses distingue le commerçant ordinaire, routinier du grand négociant qui « à l'intelligence, à l'assiduité et au goût, joint de plus cette sagacité rare, ce talent heureux d'apercevoir promptement la liaison des choses, de prévoir avec une sorte de certitude les suites des événements et de combiner exactement les probabilités de succès » (mai 1760 : 140). Et l'auteur indique que s'il est licite d'utiliser un avis particulier lorsqu'on reste dans le cadre d'une spéculation, « car toute spéculation sur des probabilités suppose de la contingence dans le succès » (ibid., p. 145), tel n'est plus le cas si le gain devient certain grâce à l'avis particulier.

24. " (...) its object is refinement, not reconstruction; it is a study in " pure theory ». The motive back of its presentation is twofold. In the first place, the writer cherishes, in the face of the pragmatic, philistine tendencies of the present age, especially caracteristic of the thought of our own country, the hope that careful, rigorous thinking in the field of social problems does after all have some significance for human weal and woe » (Knight, 1921 : vii). 
opposition à celle de Ricardo; si on considère la méthode effective mise en oeuvre, alors la différence est beaucoup moins nette, car Say est souvent aussi déductif que Ricardo. Sur le second point, un même phénomène de rapprochementdistanciation est perceptible. La structure industrielle telle que la pensait l'économiste français, dès 1803 , est caractérisée par le modèle anglais où s'effectue une « production de mêmeté ». Cela dénote une vision très claire de la production standardisée qui domine le début du $\mathrm{XX}^{\mathrm{e}}$ siècle; toutefois, les problèmes de la grande industrie prennent une dimension qu'ils ne pouvaient avoir au début du $\mathrm{XIX}^{\mathrm{e}}$ siècle et que Say n'a nullement prévue et encore moins étudiée ${ }^{25}$.

Ce mélange de différences et de rapprochements se retrouve au niveau de l'analyse économique. Knight se propose d'isoler le profit pur, reçu indépendamment de toute rémunération d'un service producteur; à cette occasion, il met l'accent sur une différence cruciale entre le risque et l'incertitude, différence qui renvoie au problème posé par le rapport des connaissances à la disposition des agents économiques et l'état de l'économie, présent et futur ${ }^{26}$. Ce faisant, Knight reprend des distinctions qui se trouvent déjà chez Say ainsi que l'étude de sa théorie de la répartition l'a montré plus haut : c'est le cas lorsque Knight caractérise l'entrepreneur comme l'agent qui affronte l'incertitude, à la différence des fournisseurs de services producteurs, et qui perçoit en conséquence un revenu incertain alors que les autres agents cèdent leurs services producteurs contre un revenu certain (Knight, 1921 : 244-245, 271); lorsqu'il souligne la différence entre la somme des prix des facteurs et le prix du produit (ibid., p. 277) ou bien lorsqu'il caractérise l'entrepreneur par le jugement (ibid., p. 211). Il est seulement curieux de constater que Knight ne fait pas de référence précise à tous ces points chez $\mathrm{Say}^{27}$.

25. Ainsi, le problème de l'entrepreneur dans la grande industrie qui occupe un chapitre entier chez Knight est quasi absent chez Say. Une des rares occurences de ce problème se trouve dans un texte de Say consacré à une compagnie pour l'approvisionnement en grains de la capitale proposée par le financier Ternaux. À cette occasion, Say indique qu'un grave problème tient au choix des administrateurs. Le fait de prévoir qu'un sixième des profits sert aux frais de gestion et à la rémunération des administrateurs est une bonne chose car outre le fait que ces frais sont plafonnés, les administrateurs sont intéressés à l'accroissement des profits qui, dans une proportion des trois quart, restent destinés aux actionnaires (Say, $1818: 44-45)$.

26. "With the introduction of uncertainty - the fact of ignorance and necessity of acting upon opinion rather than knowledge - into this Eden-Like situation, its character are completely changed. With uncertainty absent, man's energies are devoted altogether to doing things (...) With uncertainty present (...) the primary problem or function is deciding what to do and how to do it » (Knight, 1921 : 268). La présentation, très élémentaire, que nous faisons ici n'empêche pas de relever que le texte de Knight est soumis à des interprétations différentes quant à l'opposition entre le risque et l'incertitude. À ce propos, l'interprétation de R.N. Langlois et M.M. Cosgel (1993) est très intéressante lorsqu'ils contournent la question incertitude mesurable / non mesurable (ou probabilité subjective / objective) pour mettre l'accent sur le fait que, selon Knight, le jugement de l'entrepreneur porte d'abord sur la définition des situations possibles, et seulement ensuite, à la formation d'un jugement sur les probabilités afférentes à ces situations.

27. Les renvois à Say sont assez superficiels dans l'ouvrage de Knight. Par exemple, lorsque Knight (1921 : 25) mentionne la conception du profit développée par J.G. Courcelle-Seneuil, il ne relève pas le fait que ce dernier fonde explicitement ses idées sur celles de Say en citant un extrait du Traité où apparaît la distinction entre revenus certains et incertains (J.G. Courcelle-Seneuil 1852 : 455). 
Malgré tous ces rapprochements et malgré les remarques intéressantes sur la nature des incertitudes auxquelles est confronté l'entrepreneur selon Say ${ }^{28}$, il semble raisonnable de penser que l'économiste français ne s'est pas avancé exactement ainsi que Knight a été amené à le faire un siècle après lui. Deux différences marquantes doivent être soulignées à ce point : premièrement, Say n'introduit pas comme Knight (1921:267-268) la différence décisive entre la pure coordination de l'activité en univers certain et la prise de décision en univers incertain; l'entrepreneur de Say est encore associé à ces deux niveaux considérés comme deux aspects qualitativement identiques dans la définition de l'entrepreneur. Deuxièmement, lorsque Say explique que l'entrepreneur doit avoir du jugement, le rapprochement avec Knight ne doit pas masquer le fait que Say en a une définition plus générale, plus vague que celle de l'économiste américain d'une part ${ }^{29}$, mais surtout qu'il n'y a pas chez Say de développements correspondant à l'idée de Knight selon laquelle, dans la grande industrie, l'entrepreneur exerce son jugement à propos du jugement dont sont susceptibles de faire preuve les personnes agissant sous sa direction (Knight, 1921 : 287-290).

\section{CONCLUSION}

Say doit désormais prendre sa place dans la tradition théorique qui, de Cantillon à Knight, met l'accent sur la relation de l'entrepreneur à l'incertitude. S'il reprend l'opposition de Cantillon entre les revenus certains et les revenus incertains, il l'introduit dans un cadre théorique nouveau où les prix des services sont déterminés par le marché et où l'entrepreneur relie ces marchés entre eux. Par ailleurs, Say s'éloigne de la perspective de Cantillon, puisque l'indépendance lui paraît assurée pour l'entrepreneur - hors le cas un peu spécial de l'entrepreneur agricole - et que, désormais, s'il y a de la suprématie économique et sociale, c'est celle de l'entrepreneur avec tous les dangers que cela peut présenter pour le fonctionnement d'une économie de marché ${ }^{30}$. Ces caractéristiques théoriques rapprochent Say des développements de Knight de même que la distinction entre ce qui ressortit au risque (assurable) et à ce qui lui échappe.

28. Nous pensons essentiellement au fait qu'à l'occasion, Say distingue entre deux sortes d'incertitude. Il y a d'une part une incertitude calculable : c'est le cas de la relation entre le capitaliste et l'entrepreneur où ce dernier paye au premier une prime d'assurance plus ou moins forte suivant le risque que comporte l'opération; c'est aussi le cas des risques encourus par l'entrepreneur qui les assume grâce à sa prévoyance. D'autre part, il y a des risques pour lesquels l'entrepreneur affronte une incertitude radicale : « enfin il ne faut pas croire que dans les entreprises industrielles, tous les éléments de succès et de revers soient appréciables par des chiffres. On peut être secondé ou entravé par des circonstances qui ne tiennent point de l'intérêt, qui sont au-dessus du pouvoir humain et de toute prévoyance » (Mss, (H) : 393; $c f$. aussi $1814(\mathrm{H}): 118)$.

29. Dans l'ouvrage que Say - moraliste à la Chamfort - consacre au comportement humain, le jugement est défini comme la capacité à se conduire avec sagesse et par opposition aux préjugés (Say, 1817 : 51-52, 94-95); il n'y a pas chez lui d'opposition tranchée entre savoir et jugement comme chez Knight : "We act upon estimates rather than inferences, upon "judgement" or "intuition", not reasoning for the most part (Knight, 1921 : 223; cf. aussi p. 211). 1997).

30. Nous avons plus longuement développé ce point dans une autre contribution (P. Steiner, 
Les considérations qui donnent une place à Say dans la filiation CantillonKnight, ont aussi souligné l'existence de différences irréductibles entre les structures théoriques dans lesquelles prennent place les réflexions sur l'entrepreneur des trois auteurs. Il serait vain de vouloir les rapprocher en faisant fi de l'écart que creusent les siècles, avec l'évolution des structures de l'économie d'une part, avec l'évolution des questions théoriques d'autre part. Et pourtant! Personne ne peut s'empêcher de considérer avec un émerveillement mêlé de perplexité cette filiation entre trois économistes sur deux siècles, de telle manière qu'ils contribuent, chacun à sa manière, à créer un courant continu où se forment et se déforment les éléments du problème constitué par l'existence d'un agent particulier confronté aux limites du savoir sur les états futurs du monde.

\section{BIBLIOGRAPHIE}

Allix, E. (1912), « L'oeuvre économique de Germain Garnier traducteur d'Adam Smith et disciple de Cantillon », Revue d'Histoire des Doctrines Économiques et Sociale : 317-342.

BARETTO, H. (1989), The Entrepreneur in Micro-Economic Theory : Disappearance and Explanation, Routledge.

BAumol, W.J. (1968), « Entrepreneurship in Economic Theory », American Economic Review : 64-71.

Brewer, A. (1992), Richard Cantillon. Pioneer of Economic Theory, Routledge.

CANTILlon, R. (1755), Essai de la nature du commerce en général, T. TsudA (éd.), Kunokuniya (1979).

Courcelle-Seneuil, J.G. (1852), « Profit », dans C. Coquelin et C. Guillaumin (eds.) Dictionnaire de l'économie politique (II) : 454-456.

EKELUND, R.B., et R.F. HÉBERT (1990), « Alternative Visions of the Entrepreneur : Cantillon, Say, Dupuit », dans D.F. Moggridge (ed.) Perspectives on the History of Economic Thought : 117-123, Edward Elgar.

Fontaine, P. (1993), « The Capitalist Entrepreneur in Eighteenth-Century Literature », Journal of the History of Economic Thought : 72-89.

Fontaine, P., et L. MARCo, (1993), « La gestion d'entreprise dans la pensée économique française aux $18^{\mathrm{e}}$ et $19^{\mathrm{e}}$ siècles ", Revue d'économie politique : 579-598.

FRASER, L.M. (1937), Economic Thought and Language, Macmillan.

Hashimoto, H. (1980), « Notes inédites de J.-B. Say qui couvrent les marges de la Richesse des Nations et qui la critiquent », KSU Economic and Business Review : 53-81.

HeBERT, R.F., et A.N. LiNK (1988), The Entrepreneur : Mainstream Views and Radical Critiques, Praeger.

Hoselitz, B.F. (1960), " The Early History of Entrepreneurial Theory », dans J.J. SPENGLER et W.R. Allen (eds.), Essays in Economic Thought : Aristotle to Marshall : 235-258, Rand MacNally. 
Hollander, S. (1985), « On the Substantive Identity of the Ricardian and Neoclassical Conceptions of Economic Organization : The French Connection in British Classicism ", dans G.A. Caravale (ed.) The Legacy of Ricardo : 13-44, Basil Blackwell.

KIRZNER, I.M. (1973), Competition and Entrepreneurship, Chicago UP.

KNight, F.H. (1921), Risk, Uncertainty and Profits, London School of Economics (1935).

KNIGHT, F.H. (1934), « Profit », Encyclopedia of the Social Sciences, vol. 12 : 480-487, Macmillan.

KNIGHT, F.H. (1942), « Profit and the Entrepreneurial Functions », Journal of Economic History Supplement : 126-132, Journal de Commerce, Bruxelles (1759-1762).

Koolman, G. (1971), « J.-B. Say's Conception of the Role of the Entrepreneur », Economica : 269-286.

LANGLOIS, R.N., et M.M. CosGel (1993), « Frank Knight on Risk, Uncertainty, and the Firm: a New Interpretation », Economic Inquiry : 456-465.

LEROY, S.F., et L.D. Singell (1987), « Knight on Risk and Uncertainty », Journal of Political Economy: 394-406.

PEACH, T. (1993), Interpreting Ricardo, Cambridge UP.

Prendergast, R. (1991), «Cantillon and the Emergence of the Theory of Profit », History of Political Economy : 419-429.

SAY, J.-B. (Mss), Manuscrits déposés à la Bibliothèque Nationale, (Paris).

SAY, J.-B. (1803), Traité d'économie politique ou simple exposition de la manière dont se forment, se distribuent et se consomment les richesses, $\mathrm{l}^{\mathrm{re}}$ éd., Crapelet.

SAY, J.-B. (1814), Traité d'économie politique, $2^{\mathrm{e}}$ éd., Déterville.

SAY, J.-B. (1817), Petit volume contenant quelques aperçus des hommes et de la société, Déterville.

SAY, J.-B. (1818), «Lettre à Ternaux », dans TERnAUX, Mémoires sur les moyens d'assurer les subsistances de la ville de Paris par l'établissement d'une compagnie de prévoyance : 33-50, Ballard.

SAY, J.-B. (1819), Traité d'économie politique, $4^{\mathrm{e}}$ éd., Déterville.

SAY, J.-B. (1820), Lettres à Malthus sur différens sujets d'économie politique, notamment sur les causes de la stagnation générale du commerce, Bossange.

SAY, J.-B. (1821), Catéchisme d'économie politique, $2^{\mathrm{e}}$ éd., Bossange.

SAY, J.-B. (1826), Traité d'économie politique, $5^{\circ}$ éd., Calmann-Lévy (1972).

SAY, J.-B. (1828), " Discours d'ouverture du cours d'économie industrielle », dans SAY (1848), CEuvres diverses de J.-B. Say : 148-161, Guillaumin.

SAY, J.-B. (1828-1829), Cours complet d'économie politique pratique, Guillaumin et $\mathrm{C}^{\circ}$ (1852).

SchulTz, T.W. (1980), « Investment in Entrepreneurial Ability », Scandinavian Journal of Economics : 437-448. 
SCHUMPETER, J. (1911), La théorie de l'évolution économique, trad. française de la $2^{\mathrm{e}}$ éd., Dalloz (1983).

SCHUMPETER, J. (1954), History of Economic Analysis, Allen et Unwin.

Smith, A. (1776), An Inquiry into the Nature and the Cause of the Wealth of Nations, Liberty Press (1981).

STEINER, P. (1996), «L'économie politique comme science de la modernité », dans SAY (1996), Cours d'économie politique et autres essais : 9-47, GarnierFlammarion.

STEINER, P. (1997), « Politique et économie politique chez Jean-Baptiste Say », Revue Française d'Histoire des Idées Politiques, à paraitre.

STIGLER, G.S. (1941), Production and Distribution Theories, Macmillan.

TeILHAC, E. (1927), L'œuvre économique de Jean-Baptiste Say, Alcan.

VÉRIN, H. (1982), Entrepreneur, entreprise : histoire d'une idée, Puf.

Weston, F.J. (1950), « A Generalized Uncertainty Theory of Profit », American Economic Review : 40-60. 